\title{
Neue Marktforschung
}

$\mathrm{M}$

arketingerfolg hängt maßgeblich vom

Kunden- und Marktverständnis ab. Umweltveränderungen, Nachfrageschwankungen sowie eine zunehmende Marktfragmentierung fordern die Marktforschung zunehmend heraus. Verständliche Marktbeschreibungen, überzeugende Erklärungen, gute Prognosen und wirkungsvolle Handlungshinweise fallen in einer von Wandel geprägten Umwelt zunehmend schwer.

Die Ansätze von gestern helfen nur begrenzt, die Fragen von morgen zu beantworten. Mit technischen Innovationen, wie z. B. dem Internet, eröffnen sich neue Möglichkeiten zur Gewinnung von Kundendaten. Aber auch neuartige Auswertungsmethoden bieten sich an, um traditionelle Marktforschungsansätze zu erweitern.

Die vorliegende Ausgabe der Marketing Review St. Gallen befasst sich mit den zentralen Themen der „Neuen Marktforschung“. Im Mittelpunkt stehen innovative Marktforschungsansätze. Konkrete Praxisbeispiele schaffen zudem ein informatives und vielfältiges Bild dieser neuen Ansätze.

Ein erster Themenblock ist der OnlineMarktforschung gewidmet. Blogs, Communities, Foren etc. enthalten eine Fülle von Kundenwissen. Wem es gelingt, dieses Wissen für Unternehmensentscheider nutzbar zu machen, kann bestehende Marktforschungserkenntnisse komplettieren. Erfahren sie mehr über Marktforschungsmethoden im Web 2.0, den Möglichkeiten der sozialen Netzwerkanalyse und der graphischen Skalierung. Die Beiträge zu diesen Themen deuten die Zukunft der Online-Marktforschung an. Wir stehen am Anfang einer spannenden Entwicklung, die der Marketingdisziplin viele neue Impulse vermitteln kann.
Doch die Zukunft der Marktforschung findet nicht nur im Internet statt. Innovative Messverfahren können den „Werkzeugkasten der Marktforschung" wirkungsvoll ergänzen. Die Intention, die tatsächlichen Kundenbedürfnisse zu erforschen, spielt dabei eine tragende Rolle. Getragen von der Zielsetzung, Kunden in den Wertschöpfungsprozess einzubinden, entstehen neue Messverfahren, die der Innovationsentwicklung dienen. Das Bestreben, dem Kunden so nahe wie möglich zu kommen und auch latente, unbewusst ablaufende Verhaltensweisen zu verstehen, beschreiben Beiträge zu impliziten Messverfahren, Consumer Neuroscience und EyeTracking im Einzelhandel.

Auch schreitet die Entwicklung statistischer Auswertungsmethoden voran. In dieser Ausgabe beleuchten wir zwei Verfahren näher: Zum einen wird ein reduziertes Abfragedesign der Multidimensionalen Skalierung vorgestellt, zum anderen befasst sich ein Beitrag mit der Auswertung von Längsschnittdaten im Marketing. Die dynamische Perspektive der Datenauswertung erhält mit größeren und leistungsfähigeren Datenbanken eine wachsende Bedeutung.

Abschließend erhalten Sie allgemeine Managementempfehlungen zur Durchsetzung einer systematischen Kundensozialisation und Verhaltenssteuerung im Dienstleistungsbereich.

Die Möglichkeiten der Marktforschung haben sich in den vergangenen Jahren maßgeblich erweitert. Ich freue mich, in dieser Ausgabe gemeinsam mit Fachexperten aus Wissenschaft und Praxis einige Entwicklungen und Innovationen vorzustellen. Ich wünsche Ihnen eine anregende und spannende Lektüre.

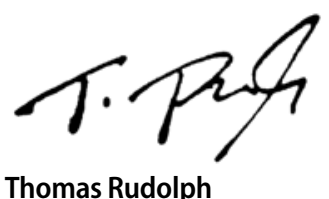

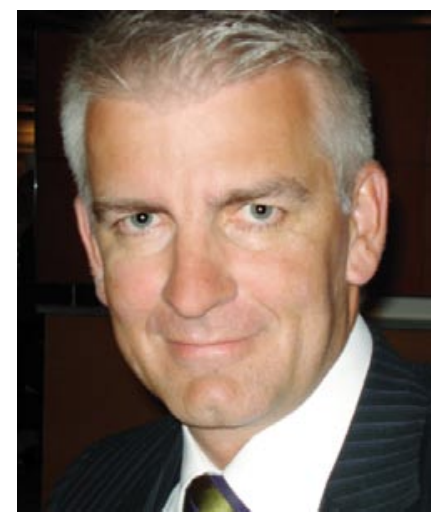

Thomas Rudolph Prof. Dr. Thomas Rudolph, Direktor am Institut für Marketing und Handel an der Universität St. Gallen und Leiter des Gottlieb Duttweiler Lehrstuhls für Internationales Handelsmanagement sowie des Retail-Labs E-Mail: thomas.rudolph@unisg.ch 